\title{
Geometrization of Electromagnetism and Gravity Based on a Finsler Space-Time with Gauge Symmetry.
}

\author{
J. P. HsU
}

Physics Department, University of Massachusetts Dartmouth

North Dartmouth, MA 02747

Mathematics Department, National Taiwan University - Taiwan

(Nuovo Cimento B, 108, 183 (1993))

PACS 04.20.Cv - Fundamental problems and general formalism.

1) The function $J_{k}^{m}$ defined for $G^{* i}{ }_{j k}=G_{j k}^{i}+g_{j m}^{i} J_{k}^{m}$ in eq. (15) needs additional conditions in order to satisfy (29). These conditions can be bypassed by a replacement $G_{j k}^{i}+g_{j m}^{i} J_{k}^{m} \rightarrow G_{j k}^{i}+\delta_{j}^{i} J_{k}(x, \dot{x})$, where $J_{k}(x, \dot{x})$ satisfies $\left(\dot{\partial}_{m} \ln U\right) \mathrm{d} \dot{x}^{m}=$ $=J_{k}(x, \dot{x}) \mathrm{d} x^{k}$. In this way, we have formally introduced $J_{k}(x, \dot{x})$ to join $\mathrm{d} \dot{x}^{m}$ and $\mathrm{d} x^{k}$ in a gauge-parallel displacement and we can derive the transformation property of $J_{k}$ to show that (27) is satisfied.

2) Since Riemann geometry is a special case of Finsler geometry, the usual physical equation of motion for a particle in an arbitrary potential field (in flat space-time) can always be derived as a geodesic equation in a curved Finsler space-time without having an external force field. Thus, the first step of the geometrization of the physical equation for particles can be done in general. However, the second step, namely, the geometrization of field equations, which determine the potential fields, is highly non-trivial. 